\title{
Two-dimensional speckle tracking echocardiography for assessment of both atria functions in patients with atrial septal defect
}

\author{
Mansour M. Mostafa, Mohammad I. Al-Deftar, Mohamed S. Mohamed* \\ Department of cardiovascular medicine, Faculty of Medicine, Al-Azhar University, Cairo, Egypt \\ *Corresponding author: Mohamed S. Mohamed; Mobile: 01000904618; Email: Se0101731@ gmail.com
}

\begin{abstract}
Background: of all the types of congenital heart disease, ASDs represent the second most common and account for $7 \%$ to $10 \%$ of all congenital heart defects. The most common type of atrial septal communication is an ostium secundum defect, representing $80 \%$ of all ASDs. In unaffected individuals, the chambers of the left side of the heart are under higher pressure than the chambers of the right side. In the case of ASD, which may result in a clinically remarkable left-to-right shunt, blood shunts from the left atrium to the right atrium. This extra blood from the left atrium may cause a volume overload of the right side.

Aim of the Work: The aim of this work was to assess feasibility of using 2-dimensional speckletracking echocardiography (2D-STE) for evaluation of left and right atrial function in patients with unrepaired atrial septal defect, to compare both LA \& RA function in patients with unrepaired atrial septal defect with those apparently healthy individuals, and to correlate both LA \& RA function with the size of inter-atrial shunting.

Patients and Methods: The ethical approval was obtained from the hospital Ethical Research Committee and each patient entering the study signed an informed consent. Thirty patients included in this study with secundum atrial septal defect. All were under the age of sixteen at time of diagnosis. They were recruited from cardiovascular department at Sayed Galal University Hospitals during the period from December 2016 to November 2018.

Results: Speckle tracking echo of both atria showed a significant correlation with QP/QS, ASD maximal size, ASD minimal size, RA to LA area ratio and pulmonary pressure. Patients with $\mathrm{PH}$ were older $(33.4+/-14.6$ vs. $23.8+/-12.2$ years; $\mathrm{p}<0.06)$ and had larger defects $(17.8+/ 4.6 \mathrm{~mm}$ vs. $14.5 .1+/-$ $5.0 \mathrm{~mm} ; \mathrm{p}<0.08$ ). The proportion of women was nearly similar in both groups (55\% in patients with $\mathrm{PH}$ vs. 63\% in those without; $\mathrm{p}=\mathrm{NS}$ ). Most patients with PH had ASDs with more complex shapes on echocardiography. However, some patients with simpler and smaller defects, such as central ASD with a diameter of $<5 \mathrm{~mm}$, also developed $\mathrm{PH}$.

Conclusion: The new echocardiographically-derived myocardial deformation indexes, which reflect structural changes assessed by transthoracic echocardiography, can be used to detect abnormalities in atrial function before clinical deterioration.
\end{abstract}

Keywords: echocardiography, atrial septal defect, right atrium, left atrium, speckle and pulmonary hypertension.

\section{INTRODUCTION}

Of all the types of congenital heart disease, ASDs represent the second most common and account for $7 \%$ to $10 \%$ of all congenital heart defects ${ }^{(1)}$. Worldwide, ASDs are noted to be 1.64 per 1000 live births with a female-to-male ratio of $2: 1^{(2)}$.

The most common type of atrial septal communication is an ostium secundum defect, representing $80 \%$ of all ASDs. Ostium primum and sinus venosus defect represent approximately $10 \%$. The rarest type of ASD is an unroofed coronary sinus ${ }^{(3)}$.

In unaffected individuals, the chambers of the left side of the heart are under higher pressure than the chambers of the right side. In the case of ASD, which may result in a clinically remarkable left-to-right shunt, blood shunts from the left atrium to the right atrium. This extra blood from the left atrium may cause a volume overload of both the right atrium and the right ventricle. If untreated, this condition can result in enlargement of the right side of the heart and ultimately heart failure ${ }^{(4)}$.

Echocardiography is the most common diagnostic method for assessing atrial function but the technique has some limitations. Traditionally, assessment of left atrial function has been performed by measuring volumes with 2D echocardiography. Additionally, it can be assessed with trans mitral Doppler and pulmonary vein Doppler ${ }^{(5)}$. 
Then, an alternative method has been incorporated, namely, measurement of myocardial deformation with color tissue Doppler derived strain. However, this method has several limitations, such as suboptimal reproducibility, angle-dependence, signal artifacts and the fact that it only measures regional strain and does not obtain information about the curved portion of the atrial $\operatorname{roof}^{(6)}$.

To overcome these limitations in the quantification of atrial function, the use of speckle tracking echocardiography (STE) strain has been proposed. This technique is not derived from Doppler but rather from 2D echocardiography; it is angle-independent and allows one to measure regional atrial strain ${ }^{(7)}$.

Two-dimensional STE has reformed cardiovascular imaging over the past decade. The methodology is based on standard B-mode images to track the motion of speckles over time and to measure the lengthening and shortening relative to the baseline value. This enables angle independent assessment of myocardial mechanics $^{(8) .}$.

However, RA not evaluated before in unrepaired ASD. Thus, we study atria functions in patients with unrepaired ASD.

Speckle tracking echocardiography is angle independent with good reproducibility ${ }^{(9)}$, and no study has been performed to assess its ability in evaluating atrial deformation.

All these characteristics make the 2D strain a tool fully compatible with the clinical scanning, able to provide additional clinical information at least ability to identify early atrial dysfunction ${ }^{(10)}$.

\section{AIM OF THE WORK}

The aim of this work was to assess feasibility of using 2-dimensional speckletracking echocardiography (2D-STE) for evaluation of left and right atrial function in patients with unrepaired atrial septal defect, to compare both LA \& RA function in patients with unrepaired atrial septal defect with those apparently healthy individuals and to correlate both LA \& RA function with the size of interatrial shunting.

\section{PATIENTS AND METHODS}

The ethical approval was obtained from the hospital ethical research committee and each patient entering the study will sign an informed consent. Thirty patients included in this study with secundum atrial septal defect. All were under the age of sixteen at time of diagnosis. They were recruited from Cardiovascular department at Sayed Galal University Hospital during the period from December 2016 to November 2018. In this study we assessed the patients by conventional echo and speckle tracking of both atria.

\section{Patients' selection}

A) Inclusion criteria (group A): These included an established diagnosis of sinus rhythm patients with isolated unrepaired secundum atrial septal defect ${ }^{(\mathbf{1})}$.

B) Exclusion criteria: Interatrial communication other than secundum ASD, Associated other congenital heart disease, Moderate or severe valvular disease, Previous cardiac surgical or percutaneous intervention, Patients with conduction abnormalities, pacemaker, ongoing arrhythmia, Documented IHD, Patients with reduced $\mathrm{EF} \leq 50 \%$, Patient refusal, concomitant severe chronic obstructive pulmonary disease or respiratory disease, or an insufficient imaging quality of the atrial endocardial border. Four patients were excluded for an insufficient image quality and 1 for the presence of atrial fibrillation.

Control group (group B): Thirty healthy individuals, age and sex matched, were used as a control group.

\section{Methods:}

(1) Clinical evaluation: included full history taking, general and local examination.

(2) Resting surface 12 ECG leads for analysis of rate, rhythm and ECG criteria of IHD.

(3) Transthoracic echocardiogram:

Based on recommendations on the echocardiographic assessment of atrial septal defect and both atria (A focused update from the European association of cardiovascular imaging and the American society of echocardiography.

A) Standard trans-thoracic echocardiographic study: LV internal dimensions, LV end-diastolic dimensions, and end-systolic dimensions, LV EF by Biplane Simpson's method, RV dimensions (basal, mid \& long), RV FAC.

B) Doppler Echocardiography: PW Doppler Mitral \& tricuspid inflow velocities (peak early diastolic velocity (E) wave, peak late diastolic velocity (A) wave, E wave deceleration time (DT), E/A ratio), By Tissue Doppler of lateral and septal mitral \& lateral 
Two-dimensional speckle tracking echocardiography for assessment of both atria functions...

tricuspid annuli (Early diastolic annulus velocity $(\mathrm{Em}), \mathrm{E} / \mathrm{Em})$.

C) ASD assessment: Maximum \& minimum diameter, PW Doppler of ASD flow, calculated the shunt fraction QP/QS.by measuring: The LVOT diameter from the PLAX view, The LVOT VTI from a 5chamber view, The RVOT diameter from the PSAX view, The RVOT VTI from the PSAX view.

D) 2D strain imaging by speckle tracking: 2D echocardiography images will be obtained from apical four chamber views. All images will be stored in cine-loop format from three consecutive beats. The frame rate for images was between 50 and 90 frames/s. All data will be transferred to a workstation for further offline analysis.

Statistical analysis: Data were tabulated and analyzed using the computer program SPSS (Statistical package for social science) version 20. Quantitative data were expressed as mean and standard deviation (SD). Qualitative data were expressed as frequency and parentage.

The following tests were done: Independentsamples t-test of significance: was used when comparing between two means. Chi-square test was used when comparing between nonparametric data.

Probability (P-value): P-value $<0.05$ was considered significant, P-value $<0.001$ was considered highly significant, $\mathrm{P}$-value $>0.05$ was considered insignificant. Receiver operating characteristics (ROC) curves: was used to detect cut-off point, sensitivity, specificity, positive predictive value (PPV) and negative predictive value (NPV). Diagnostic sensitivity: It measures the incidence of true positive results in patients' group. Diagnostic specificity: It measures the incidence of true negative results in a nondiseased group (control group). Positive predictive value: It is the percent of true positive results among all positive results. Negative predictive value: It is the percent of true negative results among all negative results.

\section{RESULTS:}

There was no statistically significant difference between patients and control as regard age $(\mathrm{P}$-value $=0.26)$. The mean age of patients was $27.3 \pm 13.7$ years while that of controls was $31.3 \pm 14$ years.

There was no statistically significant difference between patients and control as regard gender $(\mathrm{P}$-value $=0.5)$. Patients were $40 \%$ males and $60 \%$ females while controls were $43 \%$ males and $57 \%$ females.

There was no statistically significant difference between patients and control as regard family history of ASD (P-value $=0.06$ ). Patients were $86 \%$ without $\mathrm{FH}$ of ASD, and $14 \%$ with positive $\mathrm{FH}$, while controls were $100 \%$ without FH of ASD.

Table 4: Correlation between global longitudinal strain of both atria and QP/QS

\begin{tabular}{|l|l|l|l|}
\hline & variable & $\mathbf{r}$ & p-value \\
\hline \multirow{2}{*}{ Correlation } & Global longitudinal strain of both atria & \multirow{2}{*}{-0.42} & 0.02 \\
\cline { 2 - 4 } & QP/QS & & \\
\hline
\end{tabular}

This table shows statistically significant difference (P-value $<0.05)$ between global longitudinal strain of both atria and QP/QS with a moderate negative correlation between both $(\mathrm{r}=-$ 0.42 ).

Table 5: Correlation between global longitudinal strain of both atria and ASD maximal size

\begin{tabular}{|l|l|l|l|}
\hline & variable & r & p-value \\
\hline \multirow{2}{*}{ Correlation } & Global longitudinal strain of both atria & -0.44 & 0.014 \\
\cline { 2 - 4 } & ASD maximal size & \\
\hline
\end{tabular}


This table shows statistically significant difference (P-value $<0.05)$ between global longitudinal strain of both atria and ASD maximal size with a moderate negative correlation between both $(\mathrm{r}=-0.44)$.

Table 6: Correlation between global longitudinal strain of both atria and SPAP

\begin{tabular}{|l|l|l|l|}
\hline & variable & r & p-value \\
\hline \multirow{2}{*}{ Correlation } & Global longitudinal strain of both atria & -0.40 & 0.028 \\
\cline { 2 - 4 } & SPAP (mmh) & \\
\hline
\end{tabular}

This table shows statistically significant difference (P-value < 0.05) between global longitudinal strain of both atria and systolic pulmonary artery pressure with a moderate negative correlation between both $(\mathrm{r}=-0.40)$. Patients with $\mathrm{PH}$ were older $(33.4+/-14.6$ vs. $23.8+/-12.2$ years; $\mathrm{p}<0.06)$ and had larger defects $(17.8+/ 4.6 \mathrm{~mm}$ vs. $14.5 .1+/-5.0 \mathrm{~mm}$; $\mathrm{p}<0.08)$. The proportion of women was nearly similar in both groups (55\% in patients with $\mathrm{PH}$ vs. $63 \%$ in those without; $\mathrm{P}=\mathrm{NS})$. Most patients with PH had ASDs with more complex shapes on echocardiography. However, some patients with simpler and smaller defects, such as central ASD with a diameter of $<5 \mathrm{~mm}$, also developed $\mathrm{PH}$. This was in agreement with Sareban et al ${ }^{(10)}$ . the latter study included ASD group consisted of 200 patients in comparison with 643 patients with a normal echocardiogram $(36.7 \%)$ had pulmonary hypertension, defined as PASP of over $40 \mathrm{mmHg}$ (equivalent to mean pulmonary artery pressure of $25-30 \mathrm{mmHg}$ ).

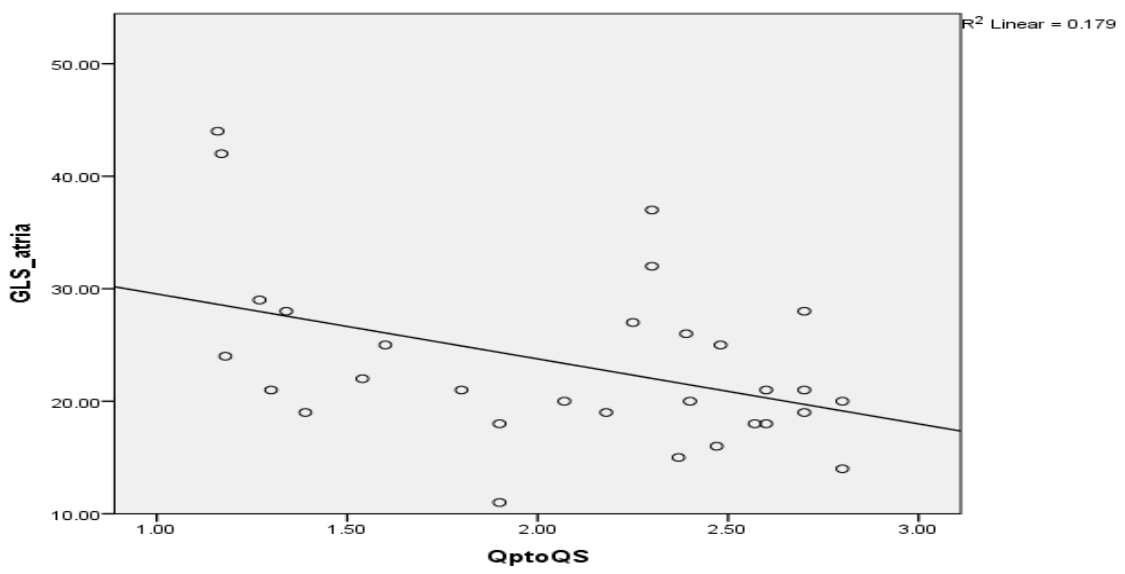

(Fig. 1): Correlation between global longitudinal strain of both atria and QP/QS

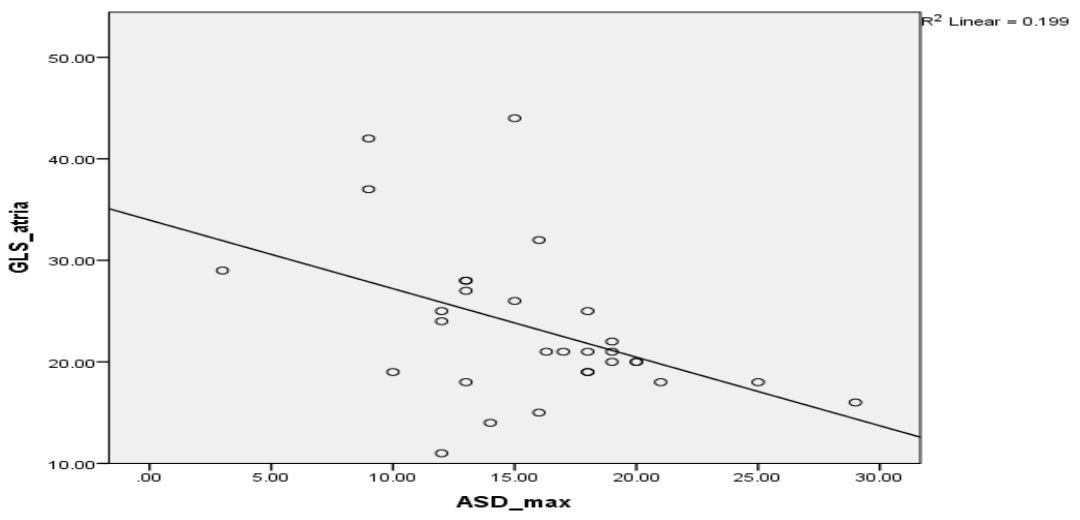

(Fig.2): Correlation between global longitudinal strain of both atria and maximal ASD size 
Two-dimensional speckle tracking echocardiography for assessment of both atria functions...

\section{DISCUSSION}

In this study, our aim was to evaluate atrial myocardial deformation properties using Doppler myocardial imaging in patients with secundum ASD. We found that atrial myocardial peak systolic strain values among the ASD group was lower than control, but this difference did not reach statistical significance.

Strain assessment as a new echo parameter has enhanced ability to assess atrial myocardial properties noninvasively. To date, this is the first study tracking the endocardium of both atria simultaneously to assess strain parameters.

The atrial function assessment by speckle tracking echocardiography is feasible with good interobserver and intraobserver variability as elaborated by this study. In the absence of other echocardiographic evidence of atrial pathology, it is useful for detection of early signs of atrial dysfunction.

In early diastole, the atria act as a conduit, which empties blood during ventricular relaxation passively, as the blood is transferred from systemic and pulmonary veins to the ventricles. Thus, atrial function during early diastole is strongly associated with ventricular compliance $^{(11)}$.

A previous study mentioned that degree of impairment in atrial compliance assessed by global longitudinal strain was a strong predictive of maintenance of sinus rhythm ${ }^{(12)}$.

During ventricular systole, the atria functions act as reservoirs to store blood when $\mathrm{AV}$ valves are closed. In this situation, reservoir function is influenced by atrial relaxation, the descent of the base to increase the negative pressure in the form of ventricle contraction, and atrial chamber stiffness. Atrial peak systolic strain is an effective measure of atrial reservoir function this is in agreement with a study done by Ancona et al. ${ }^{(2)}$

ASD causes volume overload in the right chambers of the heart due to left to right shunt, and hence increases preload and dilates these chambers. This left to right shunt in patients with an ASD leads primarily to volume overload and increases myocardial stress of the right ventricle. Consequently, right ventricular dimensions are increased in these patients. chronic volume overload results in persistent pulmonary artery pressure elevation. This was in agreement with Skhiri et al. ${ }^{(13)}$.

In contrast, left ventricular geometry is altered with mild impairment of left ventricular filling and decrease in left ventricular dimensions. After closure of the defect, right ventricular and atrial dimensions tend to decrease, but end-diastolic volume and dimensions of the left ventricle increase in the following period, and sooner return to normal ${ }^{(14)}$

It is crucial to discover the ASD patients before deterioration. The age at which symptoms appear is highly variable and is not exclusively related to the extent of shunting ${ }^{(7)}$.

The main reasons of the symptoms such as increased stroke risk and reduced exercise tolerance are right ventricular dilatation and dysfunction, arrhythmia and pulmonary hypertension.

In the case of arrhythmia, atrial flutter and atrial fibrillation cause significant morbidity, that is mainly related to the occurrence of strokes and transient ischemic attacks. It is possible that we can reduce atrial flutter and fibrillation risk by timely closure of the defect if the ASD is closed in the early stage before deterioration in atrial function. This was in agreement with Simpson et al.$^{(16)}$.

We found that atrial myocardial deformation in the form of global longitudinal strain were impaired in patients with an ASD in comparison with the control group. The atrial lengthening that occurs during ventricular ejection was reduced. These findings demonstrate the impairment of reservoir function in patients with an ASD and increased atrial stiffness. These abnormalities in the deformation properties, of the atrial myocardium which we found in patients with ASDs, maybe the result of structural changes and interstitial fibrosis, which mostly play an important role in the development of atrial arrhythmia. This was in agreement with Pathak et al ${ }^{(17)}$.

We face different cases with echo drop out at the inter atrial septum. It is challenging to make diagnosis of ASD in such group of patients without a semi-invasive or invasive procedure. This study demonstrates that speckle using to assess global longitudinal strain of both atria is helpful with good sensitivity and specificity to make the diagnosis. Our results agreed with a study done by Blume et al. ${ }^{(18)}$.

Thus, we suggest that the new echocardiographically-derived myocardial deformation indexes, which reflects structural changes assessed by transthoracic echocardiography, can be used to detect 
abnormalities in atrial function before clinical deterioration.

\section{CONCLUSION}

STE of both atria showed a significant correlation with QP/QS, ASD minimal size, RA to LA area ratio and pulmonary artery pressure. Although, atrial STE is not routinely used, its evaluation may implement atrial function assessment in secundum atrial septal defect patients.

\section{REFRENCES}

1. Wang LW, Huttner IG, Santiago CF, Kesteven SH, Yu ZY, Feneley MP and Fatkin D (2017) : Standardized echocardiographic assessment of cardiac function in normal adult zebrafish and heart disease models. Disease Models \& Mechanisms, 10(1):63-76.

2. Ancona $\mathbf{R}$, Comenale Pinto $\mathrm{S}$, Caso P, D'Andrea A, Di Salvo G, Arenga F, Coppola MG, Sellitto V, Macrino M and Calabrò $R$ (2014): Left atrium by echocardiography in clinical practice: from conventional methods to new echocardiographic techniques. Scientific World Journal, 2014:1-15.

3. Lowery KS (2016) : Atrial Septal Defects. Physician Assistant Clinics, 1(4):553562.

4. Zanchetta M, Pedon L, Rigatelli G, Carrozza A, Zennaro M, Di Martino R, Onorato E and Maiolino P (2003) : Intracardiac Echocardiography Evaluation in Secundum Atrial Septal Defect Transcatheter Closure. Cardiovascular Intervention Radiology, 26(1):52-57.

5. Rimbaș RC, Dulgheru RE and Vinereanu D (2015) : Methodological Gaps in Left Atrial Function Assessment by 2D Speckle Tracking Echocardiography. Arqous Brasilian Cardiology,105(6):625636.

6. Cianciulli TF, Saccheri MC, Lax JA, Bermann AM and Ferreiro DE (2010) : Two- dimensional speckle tracking echocardiography for the assessment of atrial function. World Journal Cardiology, 2(7):163-170.

7. Bartakian S, Fagan TE, Schaffer MS and Darst JR (2012) : Device Closure of Secundum Atrial Septal Defects in Children. JACC Cardiovasc Interv ;5(11):1178-1184

8. Ramberg E, Olausson M, Jørgensen
TBS, Nepper ML, Bhardwaj P, Binko TS, Petersen JR and Fornitz GG (2017) : Right atrial and ventricular function evaluated with speckle tracking in patients with acute pulmonary embolism. The American Journal of Emergenc Medicine, 35(1):136-143.

9. Bansal M and Kasliwal RR DE (2013) : How do I do it? Speckle-tracking echocardiography. Indian Heart Journal, 65(1):117-123.

10. Sareban M, Perz T, Macholz F, Reich B, Schmidt P, Fried S, Mairbäurl H, Berger MM and Niebauer J (2017) : Reliability of echocardiographic speckletracking derived bi-atrial strain assessment under different hemodynamic conditions. The International Journal of Cardiovascular Imaging, 33(11):16851692.

11. Mehrzad R, Rajab $M$ and Spodick DH (2017) : The three integrated phases of left atrial macro physiology and their interactions. The International Journal of Molecular Science, 15(9):15146-15160.

12.Schneider C, Malisius R, Krause K, Lampe F, Bahlmann E, Boczor S, Antz M, Ernst S and Kuck KH (2008) : Strain rate imaging for functional quantification of the left atrium: atrial deformation predicts the maintenance of sinus rhythm after catheter ablation of atrial fibrillation. European Heart Journal, 29(11):1397-1409.

13. Skhiri M, Hunt SA, Denault AY, Haddad F (2010) : Evidence-Based Management of Right Heart Failure: a Systematic Review of an Empiric Field. Revista Española de Cardiología, 63(4):451-471.

14. Raymond I, Pedersen F and SteensgaardHansen F (2003) : Prevalence of impaired left ventricular systolic function and heart failure in a middle aged and elderly urban population segment of Copenhagen. Heart, 89(12):1422-1429.

15. Van Garsse L, Gelsomino S, Luca F, Parise O, Cheriex E, Rao CM, Gensini GF and Maessen JG (2013) : Left atrial strain and strain rate before and following restrictive annuloplasty for ischaemic mitral regurgitation evaluated by twodimensional speckle tracking echocardiography. 
Two-dimensional speckle tracking echocardiography for assessment of both atria functions...

European Association of Cardiovascular Imaging, 14(6):534-543.

16. Simpson J, Lopez $L$ and Acar P (2016) : Three-dimensional echocardiography in congenital heart disease: an expert consensus document from the European Association of Cardiovascular Imaging and the American Society of Echocardiography. Eur Hear $J$ Cardiovasc Imaging ,17:1071-1097.

17. Pathak R, Lau DH, Mahajan R, Sanders $P$
(2013) : Structural and Functional Remodeling of the Left Atrium: Clinical and Therapeutic Implications for Atrial Fibrillation. J Atr Fibrillation [Internet]. CardioFront, 6(4):986- 993.

18. Blume GG, Mcleod CJ and Barnes ME (2011) : Left atrial function: physiology, assessment, and clinical implications European Association of Echocardiography, 12(6):421-430. 